\title{
La importancia de los factores internos y externos en el éxito empresarial
}

\author{
RICO BELDA, PAZ \\ Universidad de Valencia \\ Correo electrónico: prico@uv.es \\ CABRER-BORRÁS, BERNARDÍ \\ Universidad de Valencia \\ Correo electrónico: cabrerb@uv.es
}

\begin{abstract}
RESUMEN
El aumento de la productividad de una determinada economía requiere mejoras en la productividad de sus empresas, lo que se traduce en un mayor éxito empresarial. Cabe señalar que una empresa encaminada hacia el éxito es más productiva. El objetivo del trabajo es analizar cómo influyen los factores internos y los externos a la empresa en su productividad laboral. En este trabajo, se aporta evidencia que permite reconocer la importancia de la calidad de la gestión empresarial, por un lado, y de los factores relacionados con el funcionamiento de los mercados e instituciones, por otro, en la determinación de la productividad de las empresas. Los resultados evidencian que las prácticas de gestión empresarial, la calidad institucional y el entorno a la empresa contribuyen conjuntamente a la productividad empresarial. No obstante, se observan diferencias según el tamaño de las empresas, de forma que las empresas de menor tamaño presentan una menor productividad empresarial, que pueden aumentar si mejoran sus prácticas de gestión y su dotación en capital físico por trabajador. Además, si las instituciones invierten en mejorar el entorno en el que las empresas se localizan, estas verán aumentar la probabilidad de alcanzar un mayor éxito empresarial.
\end{abstract}

Palabras clave: éxito empresarial, productividad laboral, prácticas de gestión, calidad institucional.

Clasificación JEL: C13; D22; L22; M10.

MSC2010: 62H25; 62J05; 62P20; 91B69. 


\title{
The importance of internal and external factors in business success
}

\begin{abstract}
Increasing the productivity of an economy requires improvements in the productivity of its companies, and therefore greater business success. It should be noted that a company on the road to success is more productive. The objective of this paper is to analyse how internal and external factors influence the labour productivity of companies. This paper provides evidence that recognises the importance of the quality of business management, on the one hand, and the factors related to the functioning of markets and institutions, on the other, in determining the productivity of companies. The results show that business management practices and the quality of the business environment jointly determine the productivity of companies. However, there are differences according to the size of the companies, so that smaller companies have lower business productivity that can be increased if they improve their management practices and their physical capital endowment per worker. In addition, if institutions invest in improving the environment in which businesses operate, they are more likely to achieve greater business success.
\end{abstract}

Keywords: business success, labour productivity, management practices, institutional quality.

JEL classification: C13; D22; L22; M10.

MSC2010: 62H25; 62J05; 62P20; 91B69.

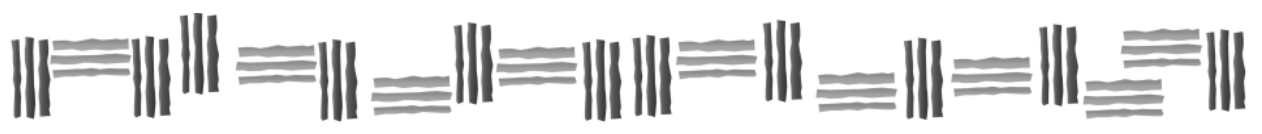




\section{Introducción.}

Está muy extendida la idea de identificar reducido tamaño empresarial con limitada productividad. Teniendo en cuenta esta idea, se considera que para conseguir aumentar la productividad hay que ampliar el tamaño empresarial. Mielgo (2015) indica que los obstáculos al crecimiento empresarial y, por tanto, al incremento de la productividad de las empresas, y así de la economía, se pueden agrupar en factores externos y en factores internos. Entre los factores externos, Mielgo considera la calidad regulatoria e institucional, la legislación fiscal y laboral y el acceso a la financiación. Aunque Mielgo no los considera, también se pueden incluir como factores externos a la empresa, y que pueden influir en su productividad, la dotación tecnológica, el capital humano y el capital emprendedor, del área geográfica donde está localizada. Respecto a los factores internos, que determinan el funcionamiento y organización de la empresa, Mielgo (2015) considera la calidad de las prácticas de gestión, el espíritu emprendedor y lo que denomina aspectos culturales, que pueden ser imperceptibles, pero que, como indica, pueden llegar a ser muy relevantes.

Huerta y Salas (2018) argumentan que, entre los economistas, está bastante extendido el convencimiento de que la reducida dimensión y la baja productividad de las empresas es consecuencia del marco regulatorio, que establece unos umbrales que dificultan el crecimiento empresarial. Como consecuencia de ello, proponen cambios en las políticas públicas que modifiquen dichos umbrales. Sin embargo, Huerta y Salas (2018) dudan de que per se la mejora de la dimensión empresarial conlleve una mejora en la productividad. Estos autores plantean la existencia de elevados costes internos de crecer, de forma que, mientras no se actué sobre ellos, se puede influir artificialmente en el tamaño de la empresa, pero sin efectos sobre la productividad, la innovación y la internacionalización, que son los factores que condicionan la competitividad. Asimismo, Huerta y Salas (2014) señalan que el tamaño de las empresas responde a factores como la inversión en formación y conocimiento, la profesionalización de la gestión, la delegación de la capacidad de decisión a niveles más bajos de la jerarquía empresarial y las relaciones cooperativas entre capital y trabajo. Por tanto, la vía para favorecer el tamaño empresarial y, finalmente, la productividad pasa por generar una mayor acumulación de capital humano y conocimiento, dentro de la empresa y también por implementar prácticas de gestión y organización empresarial más participativas. En este sentido, el empoderamiento en las empresas supone una mayor participación de sus empleados y convierte a la empresa en más productiva. En la actualidad está ampliamente reconocido que las empresas son más productivas cuando todos sus empleados tienen más capacidad de tomar decisiones y la autoridad está transferida a todos los niveles de la empresa. El modelo de gestión que busca la autonomía de los empleados conlleva ventajas, tales como una mayor motivación de los trabajadores $\mathrm{y}$, por tanto, aumentos de su productividad, mejora el ambiente de trabajo y la capacidad para resolver problemas, lo que agiliza y optimiza los procesos productivos y, en definitiva, lleva al éxito empresarial.

En el informe de la OCDE (2017, p. 61) se observa que, en algunos países, entre ellos España, Francia, Polonia y República Checa, la relación entre tamaño empresarial, medido por el número de empleados, y la productividad laboral es lineal, de forma que conforme aumenta el tamaño de las empresas aumenta su productividad. Sin embargo, en otros países, como Reino Unido y Países Bajos, la relación no es lineal y, en estos casos, se observa que la productividad laboral de las empresas grandes no es mayor que la de empresas de menor tamaño. Por tanto, no está claro que un aumento del tamaño empresarial conlleve siempre aumentos en la productividad de las empresas. Asimismo, en el mismo informe también se pone en evidencia la existencia de diferencias, entre países, en la productividad laboral de las empresas, lo que hace pensar en la existencia de factores externos que, al margen de los internos, pueden determinar la productividad empresarial.

El objetivo de este trabajo es analizar cómo influyen tanto los factores internos como los externos a las empresas en su productividad laboral. Adicionalmente, se va a agrupar la muestra de empresas, que se utiliza, según el tamaño de las mismas, con el fin comprobar si hay diferencias 
significativas en la productividad de las empresas y qué factores explicarían dichas diferencias. En concreto, se va a aportar evidencia que permite corroborar la importancia de la calidad de la gestión empresarial, por un lado, y de los factores relacionados con el funcionamiento de los mercados e instituciones, por otro, en la determinación de la productividad de las empresas. Dado que es un hecho que la gestión empresarial de calidad y un adecuado contexto institucional, donde desarrollar los negocios, conllevan buenos resultados empresariales, nuestra contribución es cuantificar, conjuntamente, la mejora generada por ambos tipos de factores y presentar la mejor estrategia para incrementar la productividad de las empresas. Habitualmente, la literatura se centra o bien en los factores externos o bien en los internos, pero no considera la repercusión conjunta de ambos factores en la productividad de las empresas. Asimismo, se analiza si existe diferencias en la productividad según el tamaño de las empresas y los factores que explicarían dichas diferencias.

Tras esta introducción, el resto del trabajo se organiza de la siguiente forma. El apartado segundo presenta el marco teórico. El tercer apartado recoge la metodología utilizada en el trabajo. El apartado cuarto presenta los datos utilizados y el quinto apartado analiza los resultados obtenidos. Finalmente, el último apartado ofrece las conclusiones y recomendaciones para aumentar la productividad de las empresas.

\section{Marco teórico.}

La gestión empresarial, como señalan Shahzad et al. (2016), conduce a la creatividad y a un adecuado desempeño organizativo y, por tanto, al éxito empresarial. Para Bloom et al. (2014), las prácticas de gestión son factores importantes para explicar la heterogeneidad y las diferencias en los resultados de las empresas. El proyecto World Management Survey (WMS) ha recopilado datos de prácticas de gestión a nivel de empresa, en diferentes sectores productivos y países (Bloom \& Van Reenen, 2007; Bloom et al., 2014). Estas prácticas de gestión son consideradas como una tecnología y, por tanto, como un input de capital intangible, que aumenta la productividad empresarial (Bloom et al., 2014; Bloom et al., 2017a).

Bloom y Van Reenen $(2007,2010)$, utilizando datos del WMS, obtienen que las empresas con mejores prácticas de gestión tienden a tener mayores resultados. Esta evidencia es confirmada por Bloom et al. (2012a), quienes emplean una muestra de la misma encuesta y por Bloom et al. (2012b), para los países en vías de desarrollo de Asia Central.

En 2010, la Oficina del Censo Americano llevó a cabo la Encuesta de Prácticas de Gestión y Organización (MOPS, por sus siglas en inglés), a más de 30.000 empresas en los Estados Unidos $y$, con datos de esta encuesta, Bloom et al. $(2013,2017 b)$ obtienen una fuerte correlación positiva entre las prácticas de gestión y el rendimiento empresarial. Por su parte, Brynjolfsson y McElheran (2016), con datos recopilados por la Oficina del Censo Americano, se centran en lo que llaman Prácticas de Toma de Decisiones basadas en Datos (DDD, por sus siglas en inglés). Estos autores encuentran que las empresas que adoptan prácticas DDD tienen rendimientos mayores que las empresas que no presentan estas prácticas.

Broszeit et al. (2016) realizan una encuesta similar a la MOPS entre empresas alemanas y muestran una sólida asociación positiva, y económicamente importante, entre las prácticas de gestión y la productividad laboral. Bryson y Forth (2018) examinan el impacto de las prácticas de gestión en el resultado empresarial en las pequeñas y medianas empresas (PYME) en Gran Bretaña, durante el período 2011-2014, y concluyen que las prácticas de gestión ayudan a las empresas a crecer y a aumentar su productividad. Bruhn et al. (2018) también confirman, para PYME empresas en México, que las prácticas de gestión tienen efectos positivos en la productividad y en los beneficios de las empresas. Rico y Cabrer-Borrás (2020a) obtienen 
evidencia de que las diferencias en la productividad laboral de las empresas y, por tanto, de los países se puede explicar, en parte, por las diferencias en las prácticas de gestión de las empresas.

Huerta y Salas (2014), utilizando un análisis de componentes principales, concluyen que la mejora de la productividad empresarial necesita de buenas prácticas de organización y gestión de las empresas, asociadas con la calidad del recurso empresarial y la delegación y cooperación entre empresario y trabajadores.

Por lo que respecta a los factores externos a la empresa, hay que considerar las características del área geográfica en la que se localiza y que tienen repercusiones en su desarrollo. Entre estas características, cabe mencionar los factores institucionales, que incluyen las normas legales y la regulación de los mercados de factores y productos, teniendo una importante repercusión la regulación del mercado laboral. Para Salmerón y Gómez (2012), el entorno institucional se define teniendo en cuenta la existencia de una serie de reglas y requisitos, a los que se someten las organizaciones, y que influyen en la gestión organizativa de éstas. Asimismo, también hay que considerar el desarrollo de los mercados financieros y el nivel de competencia y de seguridad jurídica. Finalmente, también puede influir la dotación tecnológica, el capital humano y el capital emprendedor de donde se ubica la empresa (Rico \& Cabrer-Borrás, 2020b).

La mayoría de la literatura se centra en cómo estos factores externos afectan al tamaño empresarial. Así, Kumar et al. (1999), con una muestra de 15 países europeos, concluyen que los países con sistemas judiciales eficientes tienen empresas más grandes y que las restricciones financieras limitan el tamaño empresarial. Para Huerta y Salas (2014), la competencia en los mercados y la flexibilidad del mercado laboral también tienen un efecto positivo en la dimensión empresarial.

El desarrollo de los mercados financieros y el acceso a la financiación externa por empresas y emprendedores son factores que también influyen en la actividad empresarial. Ahora bien, los factores institucionales, como el desarrollo legislativo y la eficiencia judicial, influyen como indican La Porta et al. (1997a) en el desarrollo de los mercados financieros y, por tanto, en las empresas. Asimismo, La Porta et al. (1997b) encuentran una relación positiva entre el grado de confianza generalizada de una economía, medida por el indicador que proporciona el World Values Survey, y una mayor presencia de empresas de mayor tamaño. Por su parte, Bloom y Sadun (2012) evidencian la existencia de relación entre el mismo indicador de confianza y la productividad agregada. Para Salas-Fumás et al. (2013), el efecto de la confianza en el tamaño y la productividad de las empresas se refuerza cuando se acompaña de un uso intensivo de tecnologías de la información.

El exceso o la discontinuidad de regulación, según umbrales de ingresos o tamaño, imponen obstáculos al crecimiento de la empresa y, por tanto, generan reasignaciones ineficientes en el mercado. Almunia y López-Rodríguez (2014), para España, y Garicano et al. (2013) para Francia, muestran que el endurecimiento de la regulación a partir de un determinado umbral genera la acumulación de empresas con tamaños próximos a los niveles del umbral, y que se resisten a aumentar su tamaño, para evitar un mayor control, generando ineficiencias.

\section{Metodología.}

Se parte de una de las funciones de producción más utilizadas en la literatura, la función CobbDouglas (1928), que se especifica de la siguiente forma:

$$
Y_{i}=A_{i} K_{i}^{\alpha} L_{i}^{\beta} e^{\gamma_{j} X_{i j}}
$$


donde $Y_{i}$ es la producción real de la empresa $i, A_{i}$ es la eficiencia productiva, $K_{i}$ es el stock de capital físico, $\mathrm{L}_{\mathrm{i}}$ es el trabajo y $\mathrm{X}_{\mathrm{ij}}$ es un vector de $\mathrm{j}$ factores adicionales. Además, $\alpha$ es la elasticidad de la producción para el factor capital físico y $\beta$ la elasticidad de la producción para el factor trabajo.

Dividiendo ambos miembros de la ecuación (1) por $\mathrm{L}_{\mathrm{i}} \mathrm{y}$ tomando logaritmos, la ecuación se puede reescribir a través del modelo de comportamiento siguiente:

$$
\ln \left(\frac{Y_{i}}{L_{i}}\right)=\theta+\alpha \ln \left(\frac{K_{i}}{L_{i}}\right)+(\beta+\alpha-1) \ln L_{i}+\gamma_{j} X_{i j}+\varepsilon_{i}
$$

donde la variable dependiente es el logaritmo natural de la productividad laboral y el término de eficiencia productiva $\left(\mathrm{A}_{\mathrm{i}}\right)$ se ha sustituido por una constante $(\theta)$ y por un término residual estocástico $\varepsilon_{\mathrm{i}}$.

Alternativamente, y siguiendo a Bloom et al. (2017a), se va a plantear una función de producción Cobb-Doublas para la empresa i en la que se va a incluir como factor de producción las prácticas de gestión de la empresa $\mathrm{i}\left(\mathrm{G}_{\mathrm{i}}\right)$ :

$$
Y_{i}=A_{i} K_{i}^{\alpha} L_{i}^{\beta} e^{\gamma_{j} X_{i j}} e^{\delta G_{i}}
$$

Operando como se ha hecho con la ecuación (1), la ecuación (3) se reescribe como el modelo econométrico siguiente:

$$
\ln \left(\frac{Y_{i}}{L_{i}}\right)=\theta+\alpha \ln \left(\frac{K_{i}}{L_{i}}\right)+(\beta+\alpha-1) \ln L_{i}+\gamma_{j} X_{i j}+\delta G_{i}+\varepsilon_{i}
$$

Asimismo, también se va a modelizar otro modelo en el que el término de eficiencia productiva $\left(\mathrm{A}_{\mathrm{i}}\right)$ del modelo (1) se sustituye, además de por una constante $(\theta)$ y por un término residual estocástico $\varepsilon_{\mathrm{i}}$, por un vector que incluye los $\mathrm{k}$ factores externos a la empresa $\left(\mathrm{E}_{\mathrm{k}}\right)$, quedando el siguiente modelo:

$$
\ln \left(\frac{Y_{i}}{L_{i}}\right)=\theta+\alpha \ln \left(\frac{K_{i}}{L_{i}}\right)+(\beta+\alpha-1) \ln L_{i}+\gamma_{j} X_{i j}+\varphi_{k} E_{k}+\varepsilon_{i}
$$

Finalmente, también en el modelo (3) se sustituirá la eficiencia productiva $\left(\mathrm{A}_{\mathrm{i}}\right)$ por una constante $(\theta)$, por un término residual estocástico $\varepsilon_{\mathrm{i}} \mathrm{y}$ por un vector que incluye los $\mathrm{k}$ factores externos a la empresa $\left(E_{k}\right)$, obteniéndose el modelo econométrico más completo:

$$
\ln \left(\frac{Y_{i}}{L_{i}}\right)=\theta+\alpha \ln \left(\frac{K_{i}}{L_{i}}\right)+(\beta+\alpha-1) \ln L_{i}+\gamma_{j} X_{i j}+\delta G_{i}+\varphi_{k} E_{k}+\varepsilon_{i}
$$

De esta forma, los modelos (2), (4), (5) y (6) permitirán analizar y comparar el efecto que las prácticas de gestión y los factores externos tienen en la productividad laboral de las empresas.

Varios son los factores externos considerados y cabe esperar que estén muy correlacionados, hasta el punto de plantearse cuál de ellos representa mejor el entorno donde las empresas se desarrollan. Para ello, se va a realizar un análisis de componentes principales, con el propósito de seleccionar la variable o variables con más aportación a la explicación de la varianza.

Con el objetivo de comprobar si el comportamiento de la productividad laboral de las empresas es diferente, según el tamaño empresarial, se va a segmentar la muestra aplicando al modelo (6) la descomposición de Blinder (1973) y Oaxaca (1973), distinguiendo entre empresas grandes (más de 250 trabajadores) y empresas de menor tamaño (menos de 250 trabajadores). La descomposición de Blinder y Oaxaca determina que la diferencia de productividad entre los dos grupos de empresas responde a dos componentes. El primer componente, denominado observable 
o componente característica, se debe a las diferencias en las variables explicativas de ambos grupos de empresas y el segundo, el componente inobservado, recoge las discrepancias en los parámetros entre los dos grupos de empresas. El objetivo que se persigue con el método de descomposición es determinar qué porcentaje de la diferencia de productividad se debe a las variables explicativas o características, que difieren en ambos colectivos, y qué porcentaje a las distintas repercusiones que dichas características tienen sobre la productividad laboral.

Tras la revisión de la literatura, las hipótesis propuestas son:

$\mathrm{H}_{1}$ : Las prácticas de gestión tienen una influencia positiva en la productividad laboral.

$\mathrm{H}_{2}$ : Los factores externos a la empresa afectan positivamente a su productividad laboral.

$\mathrm{H}_{3}$ : El comportamiento de las empresas es diferente según su tamaño empresarial.

\section{Datos y variables.}

En este trabajo se utiliza una muestra de empresas de seis países europeos, que incluye a Alemania, España, Francia, Grecia, Portugal, UK. Los datos proceden de diferentes bases de datos, como World Management Survey (WMS), ORBIS, Eurostat, World Economic Forum (WEF), Global Empreneurship Monitor (GEM) y el Banco Mundial.

WMS ha recopilado información de las prácticas de gestión a nivel de empresa en diferentes sectores productivos y países. La metodología de WMS se describe en detalle en Bloom y Van Reenen (2007). En concreto, la encuesta utiliza una herramienta de evaluación basada en entrevistas que define y puntúa, de 1 (peor práctica) a 5 (mejor práctica), cada una de las 18 prácticas de gestión que considera. Las prácticas de gestión se agrupan en cuatro áreas: gestión de operaciones; supervisión del rendimiento; fijación de objetivos e incentivos a los trabajadores. Como indican Bloom et al. (2012a), conceptualmente, la encuesta está relacionada con la idea de que los activos intangibles, específicos de la empresa, y los procesos organizacionales son cruciales para determinar sus resultados.

De esta base de datos, y para la encuesta del año 2014, se obtiene la información de las prácticas de gestión de la muestra de empresas utilizada. WMS también proporciona información sobre la antigüedad de la empresa, cuantificada como el período, en años, desde su establecimiento hasta que tiene lugar la encuesta. Asimismo, también facilita una variable que toma valor uno si la empresa es una multinacional.

La base de datos ORBIS ofrece información sobre las cuentas económicas y financieras de las empresas consideradas en la muestra. Después de descartar las observaciones para las que no se tiene información, la muestra consta de 774 observaciones. Para cada una de las empresas de la muestra, ORBIS facilita información sobre el número de empleados, el volumen de activos, las ventas, la rentabilidad y el sector productivo al que pertenece la actividad de la empresa. El activo de las empresas se utiliza como proxy del capital físico en la función de producción. La rentabilidad se mide a través del rendimiento del capital empleado (ROCE), que se define como el cociente entre el beneficio antes de intereses e impuestos y el capital total empleado. La variable productividad laboral, utilizada en el modelo, se obtiene como el logaritmo de las ventas anuales por empleado.

Eurostat proporciona información sobre los gastos de I+D por PIB de cada país y WEF (2015) publica el Índice de Capital Humano (KH). Este índice evalúa el nivel de educación, habilidades y empleo de la población de cada país. Asimismo, WEF (Schwab, 2015) facilita indicadores de calidad de las instituciones públicas, en concreto el Índice de Competitividad 
Global (ICG), que incluye Requerimientos Básicos (RB), Eficiencia (EF) y Factores de Innovación y Sofisticación (FIS). Por su parte, el indicador de la Tasa de Actividad Emprendedora (TAE) se obtiene de GEM. TAE cuantifica el porcentaje de la población en edad de trabajar, que está involucrada en el proceso de iniciar una actividad empresarial, o ya es propietaria-gerente de empresas con menos de tres años y medio de antigüedad. Este estudio utiliza la TAE de oportunidad, que incluye solo los emprendedores de oportunidad, excluyendo los emprendedores de necesidad.

Finalmente, el Banco Mundial (2015), a través de los indicadores Doing Business, proporciona información sobre la realidad reguladora de los países. Tres indicadores se han considerado: el índice Doing Business (DB), el índice del crédito (Crédito) y el índice de insolvencia (Insolvencia).

Como se puede observar en la Tabla 1 el valor medio de la gestión empresarial es mayor en las empresas de más de 250 empleados. Asimismo, los valores medios de todas las medidas que recogen los factores externos también son más elevados en estas empresas. Esto está en consonancia con lo que evidenciaban La Porta et al. (1997b). Dado que los factores externos dependen del país donde se ubica la empresa, lo que está indicando esta evidencia es que o bien las empresas más grandes se localizan donde los factores externos son más favorables y, por tanto, es un factor de localización de las empresas, o bien que los factores externos fomentan el crecimiento de las empresas. Por ello, si tanto los factores internos como los externos afectan positivamente a la productividad empresarial, cabe esperar que ésta sea mayor en las empresas grandes. Lo que está por determinar es qué factores contribuyen en mayor cuantía en la productividad de las empresas y, por tanto, cuál es la mejor estrategia para incrementarla.

Tabla 1. Media de las variables.

\begin{tabular}{|c|c|c|c|c|c|c|c|c|}
\hline & $\begin{array}{c}\text { Número de } \\
\text { empresas }\end{array}$ & $\begin{array}{c}\text { Prácticas } \\
\text { de } \\
\text { gestión }\end{array}$ & Empleados* & $\begin{array}{l}\text { Ventas por } \\
\text { empleado* }\end{array}$ & Antigüedad* & Multinacional & I\&D & KH \\
\hline \multirow{3}{*}{$\begin{array}{l}\text { Total } \\
\text { Menos de } 250 \\
\text { empleados } \\
\text { Más de } 250 \\
\text { empleados }\end{array}$} & 774 & 2,84 & 160 & 182,69 & 33,00 & 0,39 & 1,39 & 33,37 \\
\hline & 541 & 2,71 & 118 & 165,32 & 32,00 & 0,30 & 1,34 & 32,83 \\
\hline & 233 & 3,15 & 467 & 230,06 & 46,37 & 0,58 & 1,51 & 34,63 \\
\hline & ICG & $\mathrm{RB}$ & EF & FIS & DB & Crédito & Insolvencia & TAE \\
\hline Total & 4,70 & 5,06 & 4,77 & 4,36 & 76,11 & 57,36 & 71,15 & 71,24 \\
\hline $\begin{array}{l}\text { Menos de } 250 \\
\text { empleados } \\
\text { Más de } 250\end{array}$ & 4,64 & 5,01 & 4,75 & 4,27 & 75,48 & 56,00 & 70,06 & 70,16 \\
\hline empleados & 4,87 & 5,18 & 4,92 & 4,57 & 77,56 & 60,54 & 73,68 & 73,76 \\
\hline
\end{tabular}

* Es la mediana de la muestra.

$\mathrm{I} \& \mathrm{D}=$ Gastos de I+D sobre PIB; $\mathrm{KH}=$ =́ndice de capital humano; $\mathrm{ICG}=$ Índice de competitividad global; RB=Requerimientos básicos; EF=Eficiencia; FIS= Factores de Innovación y Sofisticación; DB= Índice Doing Business; Crédito= Índice de crédito; Insolvencia $=$ Índice de insolvencia; $\mathrm{TAE}=$ Tasa de actividad emprendedora.

Fuente: WEF (2015), Banco Mundial, GEM y WMS.

\section{Resultados.}

Entre los índices que recogen la calidad institucional y el nivel de dotación tecnológica, de los países en los que se ubica cada una de las empresas, existe una elevada colinealidad, impidiendo introducir simultáneamente todas las variables o factores en los modelos econométricos (Tabla 
2). Asimismo, esta elevada correlación indicaría que se puede utilizar, indistintamente, cualquiera de esas variables, como representante de los factores externos, para analizar los determinantes de la productividad de las empresas. Con el fin de eliminar redundancias, del conjunto de variables representativas de los factores externos, se realiza un análisis de componentes principales, que permite seleccionar la dimensión necesaria al captar la información contenida en las citadas variables.

Tabla 2. Matriz de coeficientes de correlación simple entre variables explicativas.

\begin{tabular}{lccccccccc} 
& RB & ICG & EF & FIS & DB & Crédito & Insolvencia & I\&D & KH \\
\cline { 2 - 10 } ICG & 0,98 & 1 & & & & & & & \\
EF & 0,95 & 0,99 & 1 & & & & & & \\
FIS & 0,98 & 0,99 & 0,97 & 1 & & & & & \\
DB & 0,88 & 0,92 & 0,95 & 0,88 & 1 & & & & \\
Crédito & 0,79 & 0,86 & 0,89 & 0,82 & 0,90 & 1 & & & \\
Insolvencia & 0,87 & 0,83 & 0,98 & 0,79 & 0,90 & 0,72 & 1 & & \\
I\&D & 0,92 & 0,84 & 0,76 & 0,87 & 0,63 & 0,73 & 0,53 & 1 & \\
KH & 0,55 & 0,66 & 0,75 & 0,61 & 0,73 & 0,40 & 0,82 & 0,24 & 1 \\
TAE & 0,88 & 0,94 & 0,94 & 0,94 & 0,85 & 0,69 & 0,72 & 0,74 & 0,67 \\
\hline ICG=Índice de competitividad global; RB=Requerimientos básicos; EF=Eficiencia; & \\
FIS= Factores de Innovación y Sofisticación; DB= Índice Doing Business; & & \\
Crédito= İndice de crédito; Insolvencia= Índice de insolvencia; & & \\
I\&D=Gastos de I+D sobre PIB; KH=Índice de capital humano; & \\
TAE= Tasa de actividad emprendedora. & \multicolumn{7}{c}{ Fuente: Elaboración propia. }
\end{tabular}

La Tabla 3 recoge el análisis de componentes principales y, como se puede observar, el primer componente explica el $83,03 \%$ de la varianza total, mientras que el segundo componente explica el 10,11\%, de tal forma que los dos componentes explican, conjuntamente, más del $93 \%$ de la varianza total. No obstante, los coeficientes del primer componente indican que no hay ningún factor predominante, por lo que se puede concluir que cualquiera de ellos puede servir para representar a los factores externos.

La Tabla 4 recoge los resultados de las estimaciones realizadas, todas ellas utilizando el método de mínimos cuadrados propuesto por White (1980), con el fin de obtener estimadores consistentes bajo la existencia de heterocedasticidad. La columna (1) de la Tabla 4 recoge el modelo (2), que denominamos modelo básico. Al modelo básico se ha ido añadiendo, de forma sucesiva, las variables que recogen las prácticas de gestión y los factores externos. De esta forma, se puede analizar y comparar la contribución de los distintos factores a la productividad de las empresas. La columna (2) recoge el modelo (4), que es el modelo básico al que se le ha añadido las prácticas de gestión. Se puede observar que las prácticas de gestión, como factor interno a la empresa es significativo, lo que demostraría el cumplimiento de la $\mathrm{H}_{1}$. Las siguientes columnas, de la (3) a la (12), recogen la estimación del modelo (5), para distintos factores externos a la empresa. Como se puede observar, todos los factores externos resultan ser estadísticamente significativos, evidenciando el cumplimiento de la $\mathrm{H}_{2}$. Lo que también se puede comprobar es que los factores externos a la empresa mejoran, en mayor cuantía que las prácticas de gestión, la bondad de ajuste, en comparación con el modelo básico. De los factores externos, el que conlleva una mejor bondad de ajuste es el índice de requerimientos básicos (RB). No obstante, cabe señalar que no existen diferencias relevantes entre los factores externos, corroborando los resultados obtenidos a través del análisis de componentes principales (Tabla 3). 
Tabla 3. Análisis de componentes principales de los factores externos.

\begin{tabular}{|c|c|c|c|c|c|c|}
\hline \multicolumn{4}{|c|}{ Varianza total explicada } & \multicolumn{3}{|c|}{ Matriz de componentes } \\
\hline \multirow{2}{*}{ Componentes } & \multicolumn{3}{|c|}{ Autovalores iniciales } & \multirow[b]{2}{*}{ Variables } & \multicolumn{2}{|c|}{ Componentes } \\
\hline & Total & $\%$ varianza & $\%$ acumulado & & 1 & 2 \\
\hline 1 & 7,31 & 81,27 & 81,27 & $\mathrm{RB}$ & 0,36 & $-0,22$ \\
\hline 2 & 1,01 & 11,20 & 92,46 & $\mathrm{EF}$ & 0,37 & 0,07 \\
\hline 3 & 0,41 & 4,58 & 97,04 & FIS & 0,36 & $-0,13$ \\
\hline 4 & 0,19 & 2,08 & 99,12 & DB & 0,35 & 0,14 \\
\hline 5 & 0,08 & 0,88 & 100 & Crédito & 0,33 & 0,34 \\
\hline 6 & 0,00 & 0,00 & 100 & Insolvencia & 0,32 & $-0,23$ \\
\hline 7 & 0,00 & 0,00 & 100 & $\mathrm{I} \& \mathrm{D}$ & 0,30 & $-0,54$ \\
\hline 8 & 0,00 & 0,00 & 100 & $\mathrm{KH}$ & 0,26 & 0,67 \\
\hline 9 & 0,00 & 0,00 & 100 & TEA & 0,34 & 0,00 \\
\hline
\end{tabular}

Nota: Ver acrónimos en Tabla 2.

Fuente: Elaboración propia.

Con el objetivo de poder comparar los coeficientes estimados de las prácticas de gestión y del índice de requerimientos básicos (se selecciona este índice ya que, como se ha comentado, es el factor externo que conlleva mayor bondad de ajuste) se ha tenido en cuenta, como propone Novales (2010), el tamaño medio de la variación de cada una de las variables, medido por sus respectivas desviaciones típicas. De esta forma, se obtiene que al aumentar en una unidad las prácticas de gestión y el índice de requerimientos básicos, se produce una variación en la productividad del 7,6\% y 26,8\%, respectivamente. Estos resultados indicarían que los factores externos tienen un efecto mayor en la productividad de las empresas. A modo de resumen, se puede concluir que se evidencia el cumplimiento de las hipótesis $\mathrm{H}_{1}$ y $\mathrm{H}_{2}$, pero para la productividad resultan más relevantes los factores externos, que la gestión empresarial interna.

A continuación, se estima el modelo (6), que resulta de añadir al modelo básico las prácticas de gestión y, como factor externo, el índice de RB (Tabla 5). Asimismo, y con el fin de verificar la $\mathrm{H}_{3}$, se considera la posibilidad de que la productividad de las empresas tenga distinto comportamiento según el tamaño de las mismas. Por esta razón, se estima el modelo (6) para dos grupos de empresas, las que tienen más de 250 trabajadores y las que tienen menos de 250 trabajadores. Los resultados del modelo se presentan en la Tabla 5. Al diferenciar según el tamaño de la empresa, se observa que las prácticas de gestión afectan a las empresas grandes, pero no a las empresas de menor tamaño. Esto está en consonancia con los resultados encontrados por Bloom et al. (2010), quienes concluyen que las prácticas de gestión son, particularmente, importantes en empresas grandes, que son operacionalmente complejas, y que requieren, por tanto, de prácticas formales de gestión, que no pueden realizar las empresas más pequeñas. Por lo que respecta a las características del área geográfica en la que se desarrolla la actividad de la empresa, se observa que los factores externos afectan en mayor cuantía a las empresas de menor tamaño que a las grandes (el efecto de una variación unitaria de RB es $28,9 \%$ y $18,3 \%$, respectivamente). 
Tabla 4. Estimación de la productividad laboral.

\begin{tabular}{|c|c|c|c|c|c|c|c|c|c|c|c|c|}
\hline & $\begin{array}{c}\text { Básico } \\
(1)\end{array}$ & $\begin{array}{l}\text { Prácticas } \\
\text { de gestión } \\
\text { (2) }\end{array}$ & $\begin{array}{c}\text { ICG } \\
(3)\end{array}$ & $\begin{array}{l}\mathrm{RB} \\
(4) \\
\end{array}$ & $\begin{array}{l}\mathrm{EF} \\
(5) \\
\end{array}$ & $\begin{array}{l}\text { FIS } \\
(6) \\
\end{array}$ & $\begin{array}{l}\text { DB } \\
(7) \\
\end{array}$ & $\begin{array}{c}\text { Crédito } \\
(8)\end{array}$ & $\begin{array}{c}\text { Insolvencia } \\
(9)\end{array}$ & $\begin{array}{l}\text { I\&D } \\
(10) \\
\end{array}$ & $\begin{array}{l}\mathrm{KH} \\
(11) \\
\end{array}$ & $\begin{array}{l}\text { TAE } \\
(12) \\
\end{array}$ \\
\hline Constante & $1,337 * * *$ & $1,142 * * *$ & $-0,606 * *$ & $-1,355 * * *$ & $-0,726^{* *}$ & $-0,065$ & $-1,366 * * *$ & $-0,066$ & 0,170 & $0,670 * * *$ & $0,0535^{* *}$ & $-0,309$ \\
\hline Activo por trabajador $(\ln )$ & $0,582 * * *$ & $0,576^{* * *}$ & $0,631 * * *$ & $0,631 * * *$ & $0,628 * * *$ & $0,631 * * *$ & $0,616 * * *$ & $0,631 * * *$ & $0,610 * * *$ & $0,626^{* * *}$ & $0,600 * * *$ & $0,628 * * *$ \\
\hline Empleo $(\ln )$ & $0,107 * * *$ & $0,084 * * *$ & $0,076 * * *$ & $0,076 * * *$ & $0,079 * * *$ & $0,075 * * *$ & $0,086^{* * *}$ & $0,075 * * *$ & $0,089 * * *$ & $0,080 * * *$ & $0,098 * * *$ & $0,083 * * *$ \\
\hline Antigüedad & $0,001^{*}$ & $0,001^{*}$ & 0,001 & 0,001 & 0,001 & 1,001 & 0,001 & 0,001 & 0,001 & 0,001 & 0,001 & 0,001 \\
\hline ROCE & $0,002 * * *$ & $0,002 * *$ & $0,002 * * *$ & $0,002 * * *$ & $0,002 * * *$ & $0,002 * * *$ & $0,002 * *$ & $0,002 * * *$ & $0,002 * * *$ & $0,002 * * *$ & $0,002 * *$ & $0,002 * * *$ \\
\hline Multinacional & $0,244 * * *$ & $0,207 * * *$ & $0,167 * * *$ & $0,162 * * *$ & $0,168 * * *$ & $0,173 * * *$ & $0,173 * * *$ & $0,173 * * *$ & $0,173 * * *$ & $0,179 * * *$ & $0,206 * * *$ & $0,178 * * *$ \\
\hline Factor & - & $0,125 * * *$ & $0,389 * * *$ & $0,508 * * *$ & $0,411 * * *$ & $0,295 * * *$ & $0,035 * * *$ & $0,295 * * *$ & $0,016 * * *$ & $0,380 * * *$ & $0,023 * * *$ & $0,021 * * *$ \\
\hline Dummies industria & Sí & Sí & Sí & Sí & Sí & Sí & Sí & Sí & Sí & Sí & Sí & Sí \\
\hline R-cuadrado & 0,600 & 0,605 & 0,658 & 0,664 & 0,655 & 0,655 & 0,649 & 0,655 & 0,651 & 0,654 & 0,624 & 0,646 \\
\hline R-cuadrado ajustado & 0,589 & 0,595 & 0,649 & 0,655 & 0,646 & 0,646 & 0,640 & 0,646 & 0,642 & 0,644 & 0,614 & 0,636 \\
\hline Criterio de Akaike & 1,539 & 1,529 & 1,386 & 1,369 & 1,393 & 1,395 & 1,411 & 1,395 & 1,406 & 1,398 & 1,480 & 1,421 \\
\hline Número de observaciones & 774 & 774 & 774 & 774 & 774 & 774 & 774 & 774 & 774 & 774 & 774 & 774 \\
\hline
\end{tabular}

Nota: *** y * denotan significatividad al $1 \%$ y $10 \%$, respectivamente.

La estimación es consistente con la existencia de heterocedasticidad en la muestra

ICG=Índice de competitividad global; RB=Requerimientos básicos; EF=Eficiencia; FIS= Factores de Innovación y Sofisticación; DB= Índice Doing Business; Crédito= Índice de crédito; Insolvencia= Índice de insolvencia; $\mathrm{I} \& \mathrm{D}=$ Gastos de $\mathrm{I}+\mathrm{D}$ sobre PIB; $\mathrm{KH}=$ Índice de capital humano; $\mathrm{TAE}=$ Tasa de actividad emprendedora

Fuente: Elaboración propia. 
Tabla 5. Estimación de la productividad por tamaño de empresa.

\begin{tabular}{|c|c|c|c|}
\hline & & Empresas & Empresas \\
\hline & Total & $>250$ trabajadores & $<250$ trabajadores \\
\hline Constante & $-1,444 * * *$ & $-1,485^{* * *}$ & $-0,949$ \\
\hline Activo por trabajador $(\ln )$ & $0,627 * * *$ & $0,587 * * *$ & $0,691 * * *$ \\
\hline Empleo $(\ln )$ & $0,060 * * *$ & 0,09 & 0,001 \\
\hline Antigüedad & 0,001 & 0,001 & 0,001 \\
\hline ROCE & $0,002 * * *$ & $0,002 * *$ & 0,001 \\
\hline Multinacional & $0,137 * * *$ & $0,213 * * *$ & $-0,029$ \\
\hline Prácticas de gestión & $0,088 * *$ & 0,045 & $0,190 * * *$ \\
\hline $\mathrm{RB}$ & $0,499 * * *$ & $0,544 * * *$ & $0,371 * * *$ \\
\hline Dummies industria & YES & YES & YES \\
\hline R-cuadrado & 0,666 & 0,664 & 0,686 \\
\hline R-cuadrado ajustado & 0,657 & 0,650 & 0,660 \\
\hline Criterio de Akaike & 1,364 & 1,365 & 1,337 \\
\hline Número de observaciones & 774 & 233 & 541 \\
\hline
\end{tabular}

$\mathrm{H}_{0}$ : Igualdad de parámetros entre los dos grupos de empresas p_value $=0,0096$

Nota: *** y * denotan significatividad al $1 \%$ y $10 \%$, respectivamente. La estimación es consistente con la existencia de heterocedasticidad en la muestra.

Fuente: Elaboración propia.

Con el propósito de cuantificar el efecto que los factores externos e internos tienen en la diferencia de productividad de las empresas, según el tamaño de éstas, se va a aplicar la descomposición de Blinder-Oaxaca. El resultado de la descomposición, aplicada al modelo (6), proporciona el valor medio estimado de la productividad de cada grupo (en escala logarítmica) y la diferencia entre ellos. Como se puede comprobar en la Tabla 6, la productividad media de las empresas grandes es, como cabía esperar, mayor que la de las empresas pequeñas, de forma que la diferencia entre ellas es de 0,36. De esta diferencia, un $62,63 \%$ viene explicado por las diferentes características entre las empresas. El componente observado indica que si las empresas pequeñas tuvieran las mismas características que las grandes su productividad aumentaría en 0,22. Por su parte, el segundo componente, debido a las diferencias en los coeficientes, indica el cambio en la productividad de las empresas pequeñas, cuando se aplica los coeficientes de las grandes a las características de las pequeñas. Este componente también indica un aumento en la productividad de las empresas pequeñas, si éstas respondieran o se comportaran como las grandes. Estos resultados se expresan en la escala logarítmica, por lo que resulta aconsejable transformar los resultados a la escala original (miles de euros por trabajador). De esta forma, la media de la productividad de las empresas grandes es de 240,94 miles de euros por trabajador, mientras que la de las empresas pequeñas es de 168,90, lo que equivale a una diferencia del $42,65 \%$. Si las empresas pequeñas alcanzasen las características de las grandes, su productividad aumentaría un $24,92 \%$. Al desagregar ese incremento de la productividad entre los factores tendríamos que las prácticas de gestión aumentarían la productividad un $8,0 \%$, la dotación de capital físico por empleado un $10,7 \%$ y los factores externos un 5,6\%. Por tanto, en las empresas pequeñas, las prácticas de gestión y la mayor dotación de capital físico por empleado podrían jugar un papel muy importante para aumentar su productividad. 
Tabla 6. Descomposición de Blinder-Oaxaca por tamaño de empresa.

\begin{tabular}{lcccc}
\hline & \multicolumn{3}{c}{ Productividad en escala } & \\
\cline { 2 - 4 } & Logarítmica & $\%$ & Lineal & Estadístico z \\
\hline Más de 250 trabajadores & 5,48 & - & 240,94 & 104,99 \\
Menos de 250 trabajadores & 5,13 & - & 168,90 & 147,69 \\
Diferencia & 0,36 & 100,00 & 1,43 & 5,66 \\
Componente observado & 0,22 & 62,63 & 1,25 & 3,92 \\
Componente inobservado & 0,13 & 37,36 & 1,14 & 2,68 \\
\hline
\end{tabular}

Fuente: Elaboración propia.

Se puede concluir que los factores internos y los externos determinan, conjuntamente, la productividad de las empresas. En concreto, las prácticas de gestión y el entorno, en el que se desarrollan las empresas, son factores que impulsan la productividad empresarial. No obstante, se observa diferencias según el tamaño de las empresas, de forma que las de menor tamaño presentan una diferencia, en términos de productividad, del $42,65 \%$, respecto de las de mayor tamaño. Las diferentes características, que las empresas de menor tamaño tienen respecto a las grandes, explican el $62,63 \%$ de la diferencia en productividad. Si las empresas de menor tamaño igualaran sus características a las de las grandes su productividad aumentaría un $24,92 \%$. De este aumento, un $8,0 \%$ vendría determinado por la mejora en la gestión empresarial, un $10 \%$ por el aumento de la dotación de capital físico por trabajador y un 5,6\% explicado por el entorno.

\section{Conclusiones.}

La evidencia de los resultados obtenidos permite concluir que tanto la gestión empresarial como los factores externos, representados a través de los requerimientos básicos, afectan positivamente a la productividad laboral de las empresas. Por tanto, una mejora de la gestión empresarial y del entorno en el que se desarrollan las empresas conllevarán una mejora de la productividad laboral de éstas. No obstante, el efecto positivo de los factores externos es mucho mayor que el de la gestión empresarial, aunque hay que considerar que, a igualdad de condiciones del entorno, una mejor gestión empresarial conllevará mejores resultados. Asimismo, hay que tener en cuenta que el tamaño empresarial influye en el comportamiento de las empresas, respecto a su productividad, puesto que su respuesta, tanto a la gestión empresarial como a los factores externos, difiere según el tamaño.

Se puede afirmar que mejores prácticas de gestión conllevan mayor eficiencia en la asignación de los recursos y, por tanto, mayores resultados empresariales. Esto se ha observado en las empresas de mayor tamaño en las que las prácticas de gestión afectan positivamente su productividad. Ahora bien, si las empresas de menor tamaño tuvieran las mismas características, en términos de prácticas de gestión, que las empresas de mayor tamaño verían incrementar su productividad. Si a esto se suma un cambio en el comportamiento de las empresas de menor tamaño, de forma que consideren la profesionalización de la gestión, como un camino para mejorar la asignación de recursos, se conseguirá un incremento adicional de su productividad y, con ello, de la economía en general. Por tanto, fomentar el crecimiento empresarial per se no será suficiente si no va acompañado de mejoras en la gestión empresarial y de un cambio en la conducta de las empresas de menor tamaño, en lo que se refiere a la profesionalización de la gestión y organización empresarial.

Los países que deseen aumentar su productividad deben considerar medidas orientadas a fomentar la profesionalización de la gestión, que conlleva una asignación más eficiente de recursos. La mayor acumulación de capital humano en los trabajadores también repercutirá en la mejora de la gestión empresarial. Asimismo, se ha de favorecer la innovación, a través de la inversión en I\&D; el capital 
humano, fomentando la educación; la financiación empresarial; y la calidad institucional, facilitando regulaciones que no introduzcan ineficiencias en el mercado y genere un entorno proclive al desarrollo de la actividad empresarial.

Una de las limitaciones del trabajo es no haber considerado la formación de los trabajadores de las empresas, ya que la base de datos ORBIS no proporciona dicha información, que puede ser un factor relevante para el desarrollo de las empresas.

\section{Agradecimientos}

Paz Rico agradece el soporte del proyecto de la Consellería d'Innovació, Universitats, Ciència i Societat Digital AICO/2020/217.

\section{Referencias}

Almunia, M., \& López-Rodríguez, D. (2014). Heterogeneous Responses to Effective Tax Enforcement: Evidence from Spanish Firms. MPRA Paper 57408. https://mpra.ub.unimuenchen.de/57408/2/MPRA_paper_57408.pdf

Banco Mundial (2015). Doing Business 2015. Going Beyong Efficiency. Comparing business regulations for domestic firms in 189 Economies, A World Bank Group Flagship Report, 12th edition, World Bank Group. Washington DC.

Blinder, A. S. (1973). Wage discrimination: reduced form and structural estimate, The Journal of Human Resources, 8(7), 436-455.

Bloom, N., \& Van Reenen, J. (2007). Measuring and explaining management practices across firms and countries. The Quarterly Journal of Economics, 122(4), 1351-1408.

Bloom, N., \& Van Reenen, J. (2010). Why do management practices differ across firms and countries? Journal of Economic Perspective, 24(1), 203-224.

Bloom, N., Mahajan, A., McKenzie, D., \& Roberts, J. (2010). American Economic Review, 100(2), 619-662

Bloom, N., \& Sadun, R. (2012). The Organization of Firms Across Countries. The Quarterly Journal of Economics, 127(4), 1663-1705.

Bloom, N., Genakos, R., Sadun, R., \& Van Reenen, J. (2012a). Management practices across firms and countries. National Bureau of Economic Research, NBER Working Paper No. 17850, Cambridge, US.

Bloom, N., Schweiger, H., \& Van Reenen, J. (2012b). The land that lean manufacturing forgot? Management practices in transition countries, Economics of Transition, 20(4), 593-635.

Bloom, N., Brynjolfsson, E., Foster, L., Jarmin, R., Saporta-Eksten, I., \& Van Reenen, J. (2013). Management in America. Center for Economic Studies, CES 13-01.

Bloom, N., Lemos, R., Sadun, R., Scur, D., \& Van Reenen, J. (2014). The new empirical economics of management. National Bureau of Economic Research, NBER Working Paper No. 20102, Cambridge, US. 
Bloom, N., Sadun, R., \& Van Reenen, J. (2017a). Management as a technology? National Bureau of Economic Research, NBER Working Paper No. 22327, Cambridge, US.

Bloom, N., Brynjolfsson, E., Foster, L., Jarmin, R., Patnaik, M., Saporta-Eksten, I., \& Van Reenen, J. (2017b). What drives differences in management? National Bureau of Economic Research, NBER Working Paper No. 23300, Cambridge, US.

Broszeit, S., Fritsch, U., Görg, H., \& Laible, M.C. (2016). Management practices and productivity in Germany. IZA Discussion Paper, No. 10370, Institute for the Study of Labor (IZA), Bonn.

Bruhn, M., Karlan, D., \& Schoar, A. (2018). The Impact of Consulting Services on Small and Medium Enterprises: Evidence from a Randomized Trial in Mexico. Journal of Political Economy, 126(2), 635-687.

Brynjolfsson, E., \& McElheran, K. (2016). Data in action: Data-driven decision making in U.S. manufacturing. Centre for Economic Studies, CES Working Paper 16-06, Washington DC.

Bryson, A., \& Forth, J. (2018). The impact of management practices on SME performance. National Institute of Economic and Social Research. NIESR Discussion Paper No. 488, London.

Cobb, C., \& Douglas, P. (1928). A theory production, The American Economic Review, 18(1), 139-165.

Garicano, L., LeLarge, C., y Van Reenen, J. (2013). Firm size distorsions and the productivity distribution: Evidence from France. National Bureau of Economic Research, NBER Working Paper, No. 18841.

Huerta, E., \& Salas, V. (2014). Tamaño de las empresas y productividad de la economía española. Un análisis exploratorio. Revista Mediterráneo Económico, 25, 167-191.

Huerta, E., \& Salas, V. (2018). Productividad y tamaño de las empresas: ¿Dónde están las palancas para el cambio? En Huerta y Moral (editores), Innovación y Competitividad: Desafios para la industria española, Capítulo XIV, Funcas, 427-462, Madrid, España.

Kumar, K., Rajan, R., \& Zingales, L. (1999). What determines firm' size? National Bureau of Economic Research, NBER Working Paper, No. 7208.

La Porta, R., López-de-Silanes, F., Shleifer, A., \& Vish, R.W. (1997a). Determinants of External Finance. The Journal of Finance, 52(3), 1131-1150.

La Porta, R., López-de-Silanes, F., \& Shleifer, A., y Vish, R.W. (1997b). Trust in Large Organizations. American Economic Review, 87(2), 333-338.

Mielgo, J.A. (2015). Una reflexión sobre el tamaño empresarial en España. Información Comercial Española, 885, 117-131.

Oaxaca, R.L. (1973). Male-female wage differentials in urban labor markets, International Economic Review, 14(3), 693-709.

OCDE (2017). Entrepreneurship at a Glance 2017, OECD Publishing, Paris, http://dx.doi.org/10.1787/entrepreneur_aag-2017-en

Rico, P., \& Cabrer-Borrás, B. (2020a). Management practices and business labour productivity. Journal of Small Business Strategy, en prensa. 
Rico, P., \& Cabrer-Borrás, B. (2020b). Intangible capital and business productivity. Economic Research-Ekonomska Istraživanja, https://doi.org/10.1080/1331677X.2019.1699139

Salas-Fumás, V., Sanchez-Asin, J.J., \& Storey, D. (2013). Occupational choice, number of entrepreneurs and output: theory and empirical evidence with Spanish data. SERIEs, 5, 1-24. https://doi.org/10.1007/s13209-013-0103-5

Salmerón, R., \& Gómez, S. (2012). Relación entre los factores institucionales y el emprendimiento: análisis mediante técnicas cuantitativas. Revista de métodos cuantitativos para la economía y la empresa, 13, 54-72.

Schwab, K. (2015). The Global Competitiveness Report 2015-2016. World Economic Forum, Geneva. http://www3.weforum.org/docs/gcr/2015-2016/Global_Competitiveness_Report_2015-2016.pdf

Shahzad, K., Bajwa, S.U., Siddiqi, A.F.I., Ahmid F., \& Raza, A. (2016). Integrating knowledge management $(\mathrm{KM})$ strategies y processes to enhance organizational creativity y performance: an empirical investigation. Journal of Modelling in Management, 11(1), 154-179.

White, H. (1980). A Heteroscedasticity Consistent Covariance Matrix Estimator and a Direct Test of Heteroscedasticity. Econometrica, 48(4), 817-838. 